\title{
High grade lymphoma in the nasopharynx presented as sudden onset of bilateral blindness S Shambhu* and M Vose
}

\author{
Address: Manchester Royal Eye Hospital, Oxford Road, Manchester, M13 9WH, United Kingdom \\ Email: S Shambhu* - siddesh@doctors.org.uk; M Vose - Markjovose@doctors.org.uk \\ * Corresponding author
}

Published: 16 March 2004

BMC Ophthalmology 2004, 4:2

This article is available from: http://www.biomedcentral.com/I47I-24I5/4/2

(c) 2004 Shambhu and Vose; licensee BioMed Central Ltd. This is an Open Access article: verbatim copying and redistribution of this article are permitted in all media for any purpose, provided this notice is preserved along with the article's original URL.

\begin{abstract}
Background: Sudden onset of bilateral blindness is rare; hysteria, cortical infarction or bilateral central retinal arterial occlusion can cause this.

Case presentation: The authors describe a single case of sudden onset bilateral blindness in a patient with nasopharyngeal carcinoma, which is unusual. Biopsy revealed a high-grade lymphoma. After treatment the patient made a complete visual recovery, with no evidence of visual sequelae and no clear reasons for this complete recovery.
\end{abstract}

Conclusion: $C T$ and MR imaging did not demonstrate any lesions invading any part of the visual pathway or even indeed the occipital cortex. High dose steroids may have reduced the mass effect of the tumour or the blindness may have been hysterical but is unlikely.

\section{Background}

Nasopharyngeal carcinoma is one of the most common cancers in eastern Asia. Usually presenting with a neck mass and blood-tinged sputum [1,2]. Headache and cranial nerve abnormalities have also been reported [3], presumably from brainstem involvement. With advanced nasopharyngeal carcinoma the occurrence of distant metastases are high [4]. Typical sites include bone, liver and lung [5]. Brain metastasis is rare [6].

\section{Case presentation}

A 49-year-old female presented with an 18-hour history of complete visual loss. The patient denied perception of light. Six weeks prior to presentation the patient had complained of nasal congestion. This was treated with nasal steroid with no improvement. There were no other symptoms prior to her presentation.
On examination, both pupils were fixed and dilated. Direct and consensual reflexes were absent (Amaurotic pupils). Fundoscopy revealed a healthy retina with no papilloedama or vessel engorgement. Further ocular examination revealed roaming eye movements but no evidence of cranial nerve involvement. Medically, the patient was very distressed and could not cooperate to full examination. Urgent CT scan revealed a large nasopharyngeal mass ( $60 \mathrm{~mm}$ diameter) extending from the level of the base of the skull to approximately $\mathrm{C} 2$. The mass also extended into the right sphenoidal sinus, left maxillary sinus and bilateral nasal cavities.

A neoplastic lesion was suspected but with such widespread parasinus involvement, infection could not be excluded. The patient was given intravenous dexamethasone $(16 \mathrm{mg}$ ) with $1.2 \mathrm{~g}$ Augmentin. Regular intravenous 
hydrocortisone (200 mg - 4 hourly) was commenced. Preparations were made for theatre and urgent drainage of the sinuses.

In theatre, a large, firm nasopharyngeal mass was noted in the left and right nasal cavities extending from the post nasal space to the posterior choanae. The mass was seen to extend down to the level of the uvula in the oral cavity. Biopsies were taken. There was no evidence of pus on sphenoidotomy.

On day one post-operatively, perception of light was denied. Both pupils were fixed and dilated. Day three post operatively the patients' visual acuity suddenly improved to $6 / 12$ and the pupil reactions returned to normal. By day four, vision had recovered to $6 / 5$ and she was able to read N5 print unaided. Goldmann visual field perimetry was completely unremarkable and the patient scored fully on Ishihara test plates.

Formal CT and MRI reports were obtained. Neither demonstrated optic nerve/chiasm compression by mass or associated fluid. The MRI report stated that several "high density areas in the occipital cortex would account for the patient's visual symptoms". Visual evoked potentials (VEP) were conducted and found to be unremarkable. However, there was no demonstrable field defect on full Goldmann visual field screening. Histology of the mass confirmed high-grade Non-Hodgkins lymphoma. Chemotherapy was commenced by the physicians and a cure was anticipated.

Choroid metastasis from nasopharyngeal carcinoma has previously been reported as a cause of visual disturbance [7]. There have been two case reports of visual loss being the initial presentation of nasopharyngeal carcinoma $[8,9]$. These cases were reported as unilateral visual loss. In either case, the CT scan demonstrated a space-occupying lesion compromising the orbital apex area and there was no improvement in vision, even after treatment [8]. A case of cortical blindness associated with nasopharyngeal carcinoma has been previously reported [10]. In this case, the patient had bilateral occipital lobe metastases causing permanent loss of vision. Blindness has also been reported as a result of chemotherapy in treating nasopharyngeal carcinoma [11].

\section{Conclusion}

The patient made a full visual recovery with no lasting visual field defect. This is testimony to the absence of optic pathway or cortical involvement and worthy of note. The mass effect of the tumour on the brain stem may explain the patient's fixed dilated pupils, but not her blindness. Invasion of the right sphenoidal sinus by the tumour may have caused a localized visual field defect, but this proved not to be so. The CT and MRI scans specifically reported no optic chiasm involvement from tumour or associated oedema. There was no evidence of occipital cortex infarct or oedema on either CT/MRI scans. The intensive highdose steroids may have reduced the mass effect of the tumour/oedema. The "blindness" may well have been "hysterical" but in the context of the pupil reactions this seems unlikely.

\section{Competing interests}

None declared.

\section{Authors' contributions}

SS was involved in the initial drafting of the manuscript. MV reviewed and modified the manuscript prior to admission. All authors read and approved the final manuscript.

\section{References}

I. Brown GC, Magargal LE: Central retinal artery obstruction and visual acuity. Ophthalmology 1982, 89:14-19.

2. Hsu MM, Tu SM: Nasopharyngeal carcinoma in Taiwan: clinical manifestations and results of therapy. Cancer 1983, 52:362-368.

3. Rao Y, Levitt S: Nasopharyngeal carcinoma. Head and Neck Cancer; Clinical Decision and Management Principles. Year Book Medical Publishers, Chicago; 1986:265-272.

4. Ahmad A, Stefani S: Distant metastases of nasopharyngeal carcinoma: a study of $\mathbf{2 5 6}$ male patients. Journal of Surgical Oncology 1986, 33:194-197.

5. Khor TH, Tan BC, Chua EJ, Chia KB: Distant metastases in nasopharyngeal carcinoma. Clin Radiol 1978, 29:27-30.

6. Ho $\mathrm{JH}$ : An epidemiologic and clinical study of nasopharyngeal carcinoma. Int J Radiat Oncol Biol Phys 1978, 4:182-198.

7. Jonathan S, Sham T, Choy D: Choroid metastasis from nasopharyngeal carcinoma. European Journal of Surgical Oncology 1991, I 7:384-387.

8. Ling-Yuh Kao, Huei-Chun Chuang, Yu-Song Liang: Visual Loss as the initial presentation of Nasopharyngeal carcinoma. Journal of Clinical Neuro-ophthalmology 1993, I 3(I):24-26.

9. Carlin L, Biller J, Laster , Toole JF: Monocular blindness in nasopharyngeal cancer. Archives of neurology I981, 38(9):600.

10. Adams RD, Victor M: Syndromes due to focal cerebral lesions. Harrison's Principle of Internal Medicine McGraw-Hill, New York; 1991:200-203.

II. Schornagel JH, Verweij J, De Mulder PH, Wildiers J, Kirkpatrick A, Cognetti F: Acute blindness during treatment of recurrent or metastatic nasopharyngeal carcinoma. European Journal of Cancer 1990, 26(7):857.

\section{Pre-publication history}

The pre-publication history for this paper can be accessed here:

http://www.biomedcentral.com/1471-2415/4/2/prepub 